\title{
Does Fixing Bug Increase Robustness in Deep Learning?
}

\author{
Rangeet Pan \\ rangeet@iastate.edu \\ Dept. of Computer Science, Iowa State University \\ 226 Atanasoff Hall, Ames, IA, USA
}

\begin{abstract}
Deep Learning (DL) based systems are utilized vastly. Developers update the code to fix the bugs in the system. How these code fixing techniques impacts the robustness of these systems has not been clear. Does fixing code increase the robustness? Do they deteriorate the learning capability of the DL based systems? To answer these questions, we studied 321 Stack Overflow posts based on a published dataset. In this study, we built a classification scheme to analyze how bug-fixes changed the robustness of the DL model and found that most of the bug-fixes can increase the robustness. We also found evidence of bug-fixing that decrease the robustness. Our preliminary result suggests that $12.5 \%$ and $2.4 \%$ of the bug-fixes in Stack Overflow posts caused the increase and the decrease of the robustness of DL models, respectively.
\end{abstract}

\section{CCS CONCEPTS}

- Software and its engineering $\rightarrow$ Software defect analysis; • Computing methodologies $\rightarrow$ Machine learning;

\section{KEYWORDS}

deep neural networks, bugs, bug fix, robustness

\section{ACM Reference Format:}

Rangeet Pan. 2020. Does Fixing Bug Increase Robustness in Deep Learning? In 42nd International Conference on Software Engineering Companion (ICSE '20 Companion), May 23-29, 2020, Seoul, Republic of Korea. ACM, New York, NY, USA, 3 pages. https://doi.org/10.1145/3377812.3382175

\section{RESEARCH PROBLEM AND MOTIVATION}

Deep Learning (DL) is popular in various domains including various safety-critical ones i.e., self-driving [6, 9, 37], aviation systems $[5,18]$, medical systems $[20,29]$, etc. Deploying an autonomous system to these safety-critical domains need a vigorous validation. In the past, we had instances, e.g., the Tesla autonomous car accident [38] that took place as the autonomous driving system failed to identify a truck and the outcome resulting in the death of the driver. Such incidents have made us question the trustworthiness of these autonomous systems. There are various researches conducted over these challenges and they are broadly categorized into two types, first, the study of the robustness property that includes

Permission to make digital or hard copies of all or part of this work for personal or classroom use is granted without fee provided that copies are not made or distributed for profit or commercial advantage and that copies bear this notice and the full citation on the first page. Copyrights for components of this work owned by others than ACM must be honored. Abstracting with credit is permitted. To copy otherwise, or republish, to post on servers or to redistribute to lists, requires prior specific permission and/or a fee. Request permissions from permissions@acm.org.

ICSE '20 Companion, May 23-29, 2020, Seoul, Republic of Korea

(c) 2020 Association for Computing Machinery.

ACM ISBN 978-1-4503-7122-3/20/05 ..\$15.00

https://doi.org/10.1145/3377812.3382175 creating synthetic vulnerabilities $[1,4,7,8]$ and building defense mechanisms [11, 22, 23, 25]. Second, studying the bugs, and corresponding fixing pattern $[12-14,43,44]$ to understand the issues faced by developers. Our study fits in the latter category.

Though prior works in understanding the ML and DNN bugs and their fixing strategies have charted the course, they do not reflect how these bug-fixes impact the robustness. Do bug fixes increase robustness? Is there any evidence of decreasing the robustness by deteriorating the learning capability of the model? To venture these research questions, we manually studied the Stack Overflow posts using a published dataset by Islam et al. [14] in a recent study.

\section{RELATED WORKS}

Study on the robustness property: In DNN models, one of the ways to measure the robustness property is by perturbating the input images that affect the decision of the models. This property is called the adversarial attack [8]. A wide spectrum of studies has been performed, and those include the generation of the adversarial examples $[4,8,16,22,23,26,36]$ and crafting defense $[4,10,11,21$, $28,34,35,42]$ against such synthetic attacks. Also, studying DNN models as software and utilizing SE techniques have been proven to be useful to validate and test the DNN model [2, 15, 17, 24, 27, 39, 40].

Study on developers' challenges: There are several studies conducted on identifying the challenges, bugs, and fixing strategies that developers faced and adopted in the deep learning domain.

Zhang et al. [44] has identified the bugs faced by the developers using the Tensorflow library and categorized them into the causes and the symptoms.

Islam et al. [12] has built the classification scheme on top of the study performed by [44] and [3] to understand the commonality and the variability of the bugs found in five deep learning libraries. The bug dataset has been utilized in their recent study [14] to understand the fixing patterns to address the bugs. This study has found a few findings that indicated that the bug fix pattern has a direct impact on the robustness of the model. While these studies have charted the course in the deep learning bugs, they do not shed light on the deeper relationship between the coding approach and the robustness of the model. Our study utilized the bug fixing dataset by [14] and study the Stack Overflow posts to understand the correlation among the bug fix patterns and the robustness.

\section{METHODOLOGY}

In this section, we discuss the classification scheme, which has been adopted to label Stack Overflow posts. While studying the Stack Overflow posts, we found three types of examples,

No Change. Fixing a bug does not affect the robustness, e.g., fixing versioning related bug that includes the changes needed to cope up with the new releases for the libraries. These bug fixing strategies do not impact the learning capabilities of the model. 
Increase. Fixing a bug that increases the robustness, e.g., adding a dropout layer to decrease the overfit that helps the DNN model to learn better.

Decrease. Fixing a bug that decreases the robustness, e.g., for matching the data format with the model input format, developers often use resize and downscale the input to fit the need. [14] has shown that it decreases the learning capability of the model and decreases the robustness of the model.

Based on the classification scheme, we labeled 321 Stack Overflow posts from the bug-fix dataset [14]. In this study, we did not execute the code; rather, we manually verified the code snippet and the discussion to understand how the changes affect the learning capability of a DNN model. Also, we referred to the root cause, bug type, and impact from the [12], which refers to the same dataset.

\section{RESULTS}

In this section, we discussed the relation between the bug-fix and the change of robustness. Our primary results suggest that $84.06 \%$ of the posts related to bug-fix do not impact the robustness. $12.50 \%$ of the Stack Overflow posts that fix a bug increases the overall robustness. However, $2.44 \%$ of the bug-fixes decrease the robustness, as discussed in Table 1. To understand the examples of the bug-fix that concerns about the robustness, we focused only on the posts with label Increase and Decrease.

Data Dimension. This genre of the bug fix regarding the shape change of the input data to align with the expectation of the model. As discussed by [14], three different types of

Table 1: Distribution of the change of robustness.

\begin{tabular}{|l|r|r|}
\hline Label & \# of Posts & \% of the Posts \\
\hline \hline No Change & 269 & $84.06 \%$ \\
Increase & 40 & $12.50 \%$ \\
Decrease & 11 & $2.44 \%$ \\
\hline
\end{tabular}

operations tune the shape of the input data, resize, reorder, and reshape. Reorder and reshape do not have an impact on the robustness. These operations changs the structure of the data, not the features. Resize can be either upscaling or downscaling. According to Xie et al. [41], upscaling an input, especially an image to a higher size and randomly adding padding, can help to decrease the effect of the adversarial attack that eventually increases the robustness. Whereas downscaling compresses the input, and the model learns fewer features than the actual input. This results in a decrease in robustness.

Loss Function. Loss function plays a crucial role in the learning process. During the backward propagation, this particular operation identifies the gap between the learned and the actual output of the DNN model. Table 2 suggests that loss function helps to increase the robustness of the DNN model. E.g., Stack Overflow post [33] states that the accuracy of the DNN model has a direct connection with the loss function, especially while using the Keras library. E.g., loss function i.e., categorical_crossentropy and binary_crossentropy chooses the accuracy metrics as categorial accuracy and binary accuracy respectively as a default choice. This posses an issue while selecting the desired choice for both loss function and accuracy metric. Choosing a wrong parameter can lead to the wrong computation of the forward and backward propagation and eventually decreases the robustness. Also, loss function can help to alleviate the effect of bias in the dataset as proposed by Lin et al. [19], where different classes of labeled data get different treatment based on the amount of bias and the proposed loss function regularizes the bias by maneuvering the loss value for the negative inputs.

Neural Connection. DNN model trains itself based on the architecture of the network provided. If there is any misconnection among the input nodes and output nodes, the model does not learn accurately. One such example in Stack Overflow post [31], where a simple dropout layer can remove the connection among two layers of the neural network that ends in achieving lower accuracy than expected. According to the implementation of the Keras dropout layer, the value can be of the range $(0,1)$. While the developer added a dropout layer with value 1 , the dropout does not perform and the model accuracy is $\sim 97 \%$. Changing the value to 0.99 that removes $99 \%$ of the edges between two layers disconnects the learning process and the resulting accuracy becomes $\sim 11 \%$ that decreases the robustness. Another way to increase the robustness for unbalanced dataset by reinitializing the weights such that the initialization punishes the classes of input having fewer examples.

Iteration. Iterating is similar to training a model robustly so that it learns the features from the input dataset more profoundly and increases the robustness. However, we have found evidence [32], where increasTable 2: Distribution of the bug-fix ing the iteration can have a negative effect by exhausting the memory or overfitting the model that ends up remembering the features rather than learning the feature and decreases the robustness.

Activation. Activation functions play a key role in the robustness by helping the model to learn features by removing the connection among nodes and edges (ReLU) or transferring the value corresponding to the nodes into the intent of the layer, i.e., prediction of the input (Softmax). However, a wrong choice can halt the training process, e.g., Stack Overflow post [30] that discusses the bug-fix related to the change of the activation function from Softmax that is primarily built for utilizing at the last layer of the classification problem. Using Softmax other than the last layer restricts the learning process and deteriorate the robustness.

\section{FUTURE WORKS AND CONCLUSION}

In this study, we manually verified the Stack Overflow posts and understand how the learning capabilities of the model changes with bug-fix. Studying the robustness property by including the effect of data bias, adversarial attacks by executing the model, and how different settings of the DNN model affect the decision taken by the DNN model, can be the extension of this study. Our preliminary study indicated that there is a direct impact on robustness with different fixing strategies. Understanding the relationships among the bug-fix strategies and the robustness can help the developers to opt for the fixing strategy that could increase the robustness. 


\section{REFERENCES}

[1] Rima Alaifari, Giovanni S Alberti, and Tandri Gauksson. 2018. Adef: An iterative algorithm to construct adversarial deformations. arXiv preprint arXiv:1804.07729 (2018).

[2] Greg Anderson, Shankara Pailoor, Isil Dillig, and Swarat Chaudhuri. 2019. Optimization and abstraction: a synergistic approach for analyzing neural network robustness. In Proceedings of the 40th ACM SIGPLAN Conference on Programming Language Design and Implementation. ACM, 731-744.

[3] Boris Beizer. 1984. Software system testing and quality assurance. Van Nostrand Reinhold Co.

[4] Nicholas Carlini and David Wagner. 2017. Towards evaluating the robustness of neural networks. In 2017 IEEE Symposium on Security and Privacy (SP). IEEE, 39-57.

[5] Taoran Cheng, Pengcheng Wen, and Yang Li. 2016. Research status of artificial neural network and its application assumption in aviation. In 2016 12th International Conference on Computational Intelligence and Security (CIS). IEEE, 407-410.

[6] Murat Dikmen and Catherine M Burns. 2016. Autonomous driving in the real world: Experiences with tesla autopilot and summon. In Proceedings of the 8th international conference on automotive user interfaces and interactive vehicular applications. ACM, 225-228.

[7] Yinpeng Dong, Fangzhou Liao, Tianyu Pang, Hang Su, Jun Zhu, Xiaolin Hu, and Jianguo Li. 2018. Boosting adversarial attacks with momentum. In Proceedings of the IEEE Conference on Computer Vision and Pattern Recognition. 9185-9193.

[8] Ian J Goodfellow, Jonathon Shlens, and Christian Szegedy. 2014. Explaining and harnessing adversarial examples. arXiv preprint arXiv:1412.6572 (2014).

[9] Nathan A Greenblatt. 2016. Self-driving cars and the law. IEEE spectrum 53, 2 (2016), 46-51.

[10] Dan Hendrycks and Kevin Gimpel. 2016. A baseline for detecting misclassified and out-of-distribution examples in neural networks. arXiv preprint arXiv:1610.02136 (2016).

[11] R Huang, B Xu, D Schuurmans, and C Szepesvári. 2015. Learning with a strong adversary. arXiv 2015. arXiv preprint arXiv:1511.03034 (2015).

[12] Md Johirul Islam, Giang Nguyen, Rangeet Pan, and Hridesh Rajan. 2019. A Comprehensive Study on Deep Learning Bug Characteristics. In Proceedings of the 2019 27th ACM Joint Meeting on European Software Engineering Conference and Symposium on the Foundations of Software Engineering (ESEC/FSE 2019). ACM, $510-520$.

[13] Md Johirul Islam, Hoan Anh Nguyen, Rangeet Pan, and Hridesh Rajan. 2019 What Do Developers Ask About ML Libraries? A Large-scale Study Using Stack Overflow. arXiv preprint arXiv:1906.11940 (2019).

[14] Md Johirul Islam, Rangeet Pan, Giang Nguyen, and Hridesh Rajan. [n. d.]. Repairing Deep Neural Networks: Fix Patterns and Challenges. In ICSE'20: The 42nd International Conference on Software Engineering.

[15] Guy Katz, Clark Barrett, David L Dill, Kyle Julian, and Mykel J Kochenderfer 2017. Reluplex: An efficient SMT solver for verifying deep neural networks. In International Conference on Computer Aided Verification. Springer, 97-117.

[16] Pavel Laskov et al. 2014. Practical evasion of a learning-based classifier: A case study. In 2014 IEEE symposium on security and privacy. IEEE, 197-211.

[17] Zenan Li, Xiaoxing Ma, Chang Xu, and Chun Cao. 2019. Structural coverage criteria for neural networks could be misleading. In Proceedings of the 41st International Conference on Software Engineering: New Ideas and Emerging Results. IEEE Press, 89-92.

[18] Kevin J Liang, Geert Heilmann, Christopher Gregory, Souleymane O Diallo, David Carlson, Gregory P Spell, John B Sigman, Kris Roe, and Lawrence Carin. 2018 Automatic threat recognition of prohibited items at aviation checkpoint with $\mathrm{x}$-ray imaging: a deep learning approach. In Anomaly Detection and Imaging with $X$-Rays (ADIX) III, Vol. 10632. International Society for Optics and Photonics, 1063203.

[19] Tsung-Yi Lin, Priya Goyal, Ross Girshick, Kaiming He, and Piotr Dollár. 2017. Focal loss for dense object detection. In Proceedings of the IEEE international conference on computer vision. 2980-2988.

[20] Geert Litjens, Thijs Kooi, Babak Ehteshami Bejnordi, Arnaud Arindra Adiyoso Setio, Francesco Ciompi, Mohsen Ghafoorian, Jeroen Awm Van Der Laak, Bram Van Ginneken, and Clara I Sánchez. 2017. A survey on deep learning in medical image analysis. Medical image analysis 42 (2017), 60-88.

[21] Xingjun Ma, Bo Li, Yisen Wang, Sarah M Erfani, Sudanthi Wijewickrema, Grant Schoenebeck, Dawn Song, Michael E Houle, and James Bailey. 2018. Characterizing adversarial subspaces using local intrinsic dimensionality. arXiv preprint arXiv:1801.02613 (2018).
[22] Aleksander Madry, Aleksandar Makelov, Ludwig Schmidt, Dimitris Tsipras, and Adrian Vladu. 2017. Towards deep learning models resistant to adversarial attacks. arXiv preprint arXiv:1706.06083 (2017).

[23] Seyed-Mohsen Moosavi-Dezfooli, Alhussein Fawzi, and Pascal Frossard. 2016 Deepfool: a simple and accurate method to fool deep neural networks. In Proceedings of the IEEE conference on computer vision and pattern recognition. 2574-2582.

[24] Rangeet Pan. 2019. Static deep neural network analysis for robustness. In Proceedings of the 2019 27th ACM Joint Meeting on European Software Engineering Conference and Symposium on the Foundations of Software Engineering. ACM, $1238-1240$.

[25] Rangeet Pan, Md Johirul Islam, Shibbir Ahmed, and Hridesh Rajan. 2019. Identifying Classes Susceptible to Adversarial Attacks. arXiv preprint arXiv:1905.13284 (2019).

[26] Nicolas Papernot, Patrick McDaniel, Somesh Jha, Matt Fredrikson, Z Berkay Celik, and Ananthram Swami. 2016. The limitations of deep learning in adversarial settings. In 2016 IEEE European Symposium on Security and Privacy (EuroS\&P). IEEE, 372-387.

[27] Kexin Pei, Yinzhi Cao, Junfeng Yang, and Suman Jana. 2017. Deepxplore: Automated whitebox testing of deep learning systems. In Proceedings of the 26th Symposium on Operating Systems Principles. ACM, 1-18.

[28] Thomas Schlegl, Philipp Seeböck, Sebastian M Waldstein, Ursula Schmidt-Erfurth, and Georg Langs. 2017. Unsupervised anomaly detection with generative adversarial networks to guide marker discovery. In International Conference on Information Processing in Medical Imaging. Springer, 146-157.

[29] Dinggang Shen, Guorong Wu, and Heung-Il Suk. 2017. Deep learning in medical image analysis. Annual review of biomedical engineering 19 (2017), 221-248.

[30] Stack Overflow. 2020. Cannot train a neural network solving XOR mapping. https://stackoverflow.com/questions/34311586.

[31] Stack Overflow. 2020. Dropout behavior in Keras with rate=1 (dropping all input units) not as expected. https://stackoverflow.com/questions/48355700.

[32] Stack Overflow. 2020. Tensorflow Allocation Memory: Allocation of 38535168 exceeds $10 \%$ of system memory. https://stackoverflow.com/questions/50304156.

[33] Stack Overflow. 2020. Why binary crossentropy and categorical crossentropy give different performances for the same problem? https://stackoverflow.com/ questions/42081257.

[34] Christian Szegedy, Wojciech Zaremba, Ilya Sutskever, Joan Bruna, Dumitru Erhan, Ian Goodfellow, and Rob Fergus. 2013. Intriguing properties of neural networks. arXiv preprint arXiv:1312.6199 (2013).

[35] Florian Tramèr, Alexey Kurakin, Nicolas Papernot, Ian Goodfellow, Dan Boneh, and Patrick McDaniel. 2017. Ensemble adversarial training: Attacks and defenses. arXiv preprint arXiv:1705.07204 (2017)

[36] Florian Tramèr, Fan Zhang, Ari Juels, Michael K Reiter, and Thomas Ristenpart. 2016. Stealing machine learning models via prediction apis. In 25 th $\{$ USENIX\} Security Symposium (\{USENIX\} Security 16). 601-618.

[37] Iis P Tussyadiah, Florian J Zach, and Jianxi Wang. 2017. Attitudes toward autonomous on demand mobility system: The case of self-driving taxi. In Information and Communication Technologies in Tourism 2017. Springer, 755-766.

[38] Bill Vlasic and Neal E Boudette. 2016. Self-driving Tesla was involved in fatal crash, US says. New York Times 302016 (2016)

[39] Tsui-Wei Weng, Huan Zhang, Hongge Chen, Zhao Song, Cho-Jui Hsieh, Duane Boning, Inderjit S Dhillon, and Luca Daniel. 2018. Towards fast computation of certified robustness for relu networks. arXiv preprint arXiv:1804.09699 (2018).

[40] Matthew Wicker, Xiaowei Huang, and Marta Kwiatkowska. 2018. Feature-guided black-box safety testing of deep neural networks. In International Conference on Tools and Algorithms for the Construction and Analysis of Systems. Springer, 408-426.

[41] Cihang Xie, Jianyu Wang, Zhishuai Zhang, Zhou Ren, and Alan Yuille. 2017. Mitigating adversarial effects through randomization. arXiv preprint arXiv:1711.01991 (2017).

[42] Ziang Yan, Yiwen Guo, and Changshui Zhang. 2018. Deep Defense: Training DNNs with Improved Adversarial Robustness. In Advances in Neural Information Processing Systems. 419-428.

[43] Tianyi Zhang, Cuiyun Gao, Lei Ma, Michael R. Lyu, and Miryung Kim. 2019. An Empirical Study of Common Challenges in Developing Deep Learning Applications. In 2019 IEEE 30th International Symposium on Software Reliability Engineering (ISSRE). IEEE.

[44] Yuhao Zhang, Yifan Chen, Shing-Chi Cheung, Yingfei Xiong, and Lu Zhang. 2018. An empirical study on TensorFlow program bugs. In Proceedings of the 27th ACM SIGSOFT International Symposium on Software Testing and Analysis. ACM, 129-140. 